\title{
ARTICLE
}

\section{Natural variation in CTB4a enhances rice adaptation to cold habitats}

Zhanying Zhang ${ }^{1, \star}$, Jinjie $\mathrm{Li}^{1, \star}$, Yinghua Pan ${ }^{1,2, \star}$, Jilong $\mathrm{Li}^{1, \star}$, Lei zhou ${ }^{1,3, \star}$, Hongli Shi ${ }^{1, \star}$, Yawen Zeng ${ }^{4}$, Haifeng Guo ${ }^{1}$, Shuming Yang ${ }^{4}$, Weiwei Zheng ${ }^{1}$, Jianping Yu${ }^{1}$, Xingming Sun ${ }^{1}$, Gangling Li ${ }^{1}$, Yanglin Ding ${ }^{5}$, Liang Ma ${ }^{5}$, Shiquan Shen ${ }^{4}$, Luyuan Dai ${ }^{4}$, Hongliang Zhang ${ }^{1}$, Shuhua Yang ${ }^{5}$, Yan Guo ${ }^{5} \&$ Zichao Li $^{1}$

Low temperature is a major factor limiting rice productivity and geographical distribution. Improved cold tolerance and expanded cultivation to high-altitude or high-latitude regions would help meet growing rice demand. Here we explored a QTL for cold tolerance and cloned the gene, CTB4a (cold tolerance at booting stage), encoding a conserved leucine-rich repeat receptor-like kinase. We show that different CTB4a alleles confer distinct levels of cold tolerance and selection for variation in the CTB4a promoter region has occurred on the basis of environmental temperature. The newly generated cold-tolerant haplotype Tej-Hap-KMXBG was retained by artificial selection during temperate japonica evolution in cold habitats for low-temperature acclimation. Moreover, CTB4a interacts with AtpB, a beta subunit of ATP synthase. Upregulation of CTB4a correlates with increased ATP synthase activity, ATP content, enhanced seed setting and improved yield under cold stress conditions. These findings suggest strategies to improve cold tolerance in crop plants.

\footnotetext{
${ }^{1}$ Key Laboratory of Crop Heterosis and Utilization, Ministry of Education/Beijing Key Laboratory of Crop Genetic Improvement, Department of Plant Genetics and Breeding, China Agricultural University, Beijing 100193, China. ${ }^{2}$ Rice Research Institute, Guangxi Academy of Agricultural Sciences, Nanning 530007, China. ${ }^{3}$ Hubei Key Laboratory of Food Crop Germplasm and Genetic Improvement, Food Crops Institute, Hubei Academy of Agricultural Sciences, Wuhan 430064, China. ${ }^{4}$ Biotechnology and Genetic Resources Institute, Yunnan Academy of Agricultural Sciences, Kunming 650205, China. ${ }^{5}$ State Key Laboratory of Plant Physiology and Biochemistry, College of Biological Sciences, China Agricultural University, Beijing 100193, China. * These authors contributed equally to this work. Correspondence and requests for materials should be addressed to Z.L. (email: lizichao@cau.edu.cn).
} 
$\mathrm{R}$ ice (Oryza sativa L.) is a staple food crop feeding over onehalf of the world population and includes two subspecies, indica and japonica, that originate from tropical or subtropical areas ${ }^{1,2}$. As rice is a cold-sensitive crop, low temperatures restricts its cultivation in cold habitats ${ }^{2}$. Compared with indica, japonica is mainly planted in higher altitude and latitude habitats. Accordingly, japonica rice is more cold tolerant than indica ${ }^{2,3}$.

Cold injury in rice affects both the vegetative (germination and seedling) and reproductive (booting and flowering) growth stages. Cold stress at the booting stage is a critical factor in rice production as it causes spikelet sterility and increased susceptibility to certain diseases 2,4 . It is a major problem for rice cultivation in 24 countries, including China, Japan and Korea 5 . Rice is widely planted in China, from Hainan island (N: $19-20^{\circ}$ ) to Mohe River $\left(\mathrm{N}: 52-53^{\circ}\right)$ in Heilongjiang, and from the eastern coastal areas to the Yunnan-Guizhou Plateau. China's annual loss of rice due to low temperatures was 3-5 million tons ${ }^{6}$. Therefore, improving cold tolerance at the booting stage is one of the most important tasks for rice breeders and it would be beneficial to develop cold-tolerant rice varieties using genes derived from existing germplasm resources.

Cold tolerance is a complex trait that is controlled by multiple loci and affected by the environment. Compared with other agronomic traits, dissecting the genetic basis of cold tolerance in rice has occurred relatively slowly. Cold tolerance of rice at different growth stages is probably controlled by different genes ${ }^{3}$. In the past decades, although many QTLs conferring cold tolerance at the vegetative or reproductive stage have been mapped on almost 12 chromosomes $^{2,4,7-20}$, only a few genes conferring cold tolerance at the vegetative growth stage have been isolated, such as COLD1, qLTG3-1 and LTG1 (refs 21-23). Only one gene $C t b 1$ (ref. 24) controlling cold tolerance at the booting stage has been cloned, and little is known about the underlying molecular mechanisms of cold tolerance at the booting stage, in part because of difficulties in accurately evaluating phenotypes and due to the complexity of the associated genetic pathways.

Yunnan province is located on the southwest plateau of China and is characterized by low average temperatures during the rice growing period (Supplementary Fig. 1a). In our previous work, many cold-tolerant landraces were identified, of which japonica variety KUNMINGXIAOBAIGU (KMXBG) was relatively tolerant of cold at both the seedling and booting stages ${ }^{25,26}$. To investigate the genetic basis of cold tolerance at the booting stage, a cross between KMXBG (cold-tolerant) and Towada (coldsensitive) was made to develop a set of cold-tolerant near-isogenic lines using KMXBG as a donor. Eight QTLs conferring cold tolerance at the booting stage were mapped on chromosomes 1,4 , 5, 10 and 11 (ref. 9).

In this study, we characterized a near-isogenic line (NIL) NIL1913 that exhibited more cold tolerance than the recurrent parent Towada and included the QTL, qCTB4-1. We established an advanced back-cross population $\mathrm{BC}_{6} \mathrm{~F}_{2}$, fine mapped and cloned the target gene, CTB4a, which encodes a leucine-rich repeat receptor-like protein kinase (LRR-RLK). This work uncovers a novel gene for cold tolerance with potential value in breeding.

\section{Results}

Characterization of NIL1913 and identification of CTB4a. In our previous work, two linked QTLs, qCTB4-1 and qCTB4-2, were mapped on the short arm of chromosome 4 . We constructed a NIL, NIL1913 with $q C T B 4-1$, hereafter referred as CTB4a, from KMXBG in a Towada genetic background. NIL1913 showed a similar morphology to Towada under normal cultivation conditions in Beijing (Fig. 1a,b and Supplementary Table 1), but had stable improved cold tolerance at the booting stage with higher seed setting and pollen fertility (Fig. 1a-d, Supplementary Table 1 and Supplementary Fig. 2) when subjected to different kinds of cold stress conditions, including cold stress in deep water (CS-DW, Supplementary Fig. 1b,c), in a phytotron (CS-PT, Supplementary Fig. 1b) and in a high-altitude area (CS-HAA, altitude $1,974 \mathrm{~m}$ ) with naturally low temperatures near Kunming (Supplementary Fig. 1a,b).

To isolate $C T B 4 a$, we carried out high resolution mapping using 3,102 $\mathrm{F}_{2}$ individuals generated from backcrossing NIL1913 with Towada. Among the 3,102 individuals, a total of 46 recombinants between RM2146 and RM6770 were selected for further work. According to the genotypic groups of markers RM2146 and RM6770, five kinds of recombinant individuals (G1-G5) were grouped (Supplementary Fig. 3a). To eliminate the effect of qCTB4-2, 25 recombinant individuals from groups G1 and G2 were analysed. The 25 recombinant individuals were divided into four groups (NA, NB, NC and ND) using RM16349, RM5414 and SSR29 (Supplementary Fig. 3b). There was no significant difference in seed setting between groups NA and NB or between groups NC and ND. However, the seed setting of groups NA and NB were much lower than that of NC and ND (Supplementary Fig. 3b). These results indicated that qCTB4-1 was located in the region between RM16349 and SSR29. Next, we developed new polymorphic markers for detecting the genotypes of total six recombinant individuals of groups NB and NC, and finally delimited the CTB4a-containing DNA fragment to a $56.8 \mathrm{~kb}$ region between markers STS5 and STS12 (Fig. 1e).

Within this genomic region, four ORFs were predicted by RGAP (Rice Genome Annotation Project; Fig. 1e). We identified the full-length cDNA corresponding to ORF4 (LOC_Os04g04330), which encodes a LRR-RLK (Supplementary Fig. 4a). Sequence analysis showed 9 SNPs and 1 Indel in the promoter region, and 6 SNPs and 1 Indel in the coding region of ORF4 between KMXBG and Towada, among which 2 SNPs (T425C, T2063C) and 1 Indel (CTC 49-51) resulted in amino acid changes (Fig. 1e). The expression of CTB4a was highly induced in panicles and leaves of NIL1913 by cold stress compared to Towada (Fig. If and Supplementary Fig. 5). We postulated that ORF4 was the probable candidate gene for CTB4a.

CTB4a confers cold tolerance. To confirm our prediction, a complementary construct containing a $3.2 \mathrm{~kb}$ promoter fragment fused with the coding region and $3^{\prime}$-UTR of CTB $4 a$ from KMXBG was introduced into Towada (Fig. 2a). Phenotypic analysis of two transgenic plants, $\mathrm{C} 11$ and $\mathrm{C} 12$, with higher CTB4a expression (Supplementary Fig. 6a), showed that the cold sensitivity of Towada was rescued. Relative seed setting of C11 and $\mathrm{C} 12$ was significantly higher than that of wild type, Towada, under cold stress (Fig. 2b). We concluded that ORF4 was the functional gene for CTB4a.

To determine whether differences in the promoter or coding regions were responsible for the function of $C T B 4 a$, several experiments were conducted. First, a vector containing pCTB4aKMXBG::GUS or pCTB4a Towada.:GUS (with the GUS reporter gene driven by the promoter of the CTB4a allele from KMXBG or the allele from Towada) was introduced into Nipponbare (Nip). Expression analysis showed that the promoter of the CTB4a allele from KMXBG showed higher GUS activity than that from Towada under cold stress at the booting stage (Supplementary Fig. 7). Then two overexpression constructs containing the CTB4a allele from KMXBG (K-OE) or from Towada (T-OE) driven by the $35 \mathrm{~S}$ promoter from tobacco cauliflower mosaic virus (CaMV35S) were separately introduced into Towada (Fig. 2a). The K-OE1 and K-OE12 lines showed 


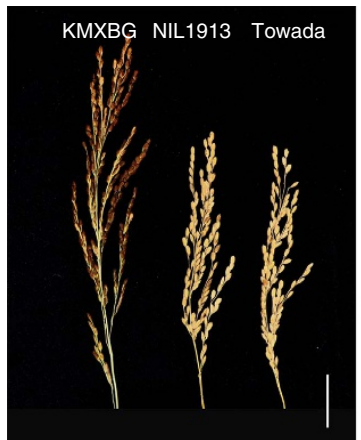

Normal conditions

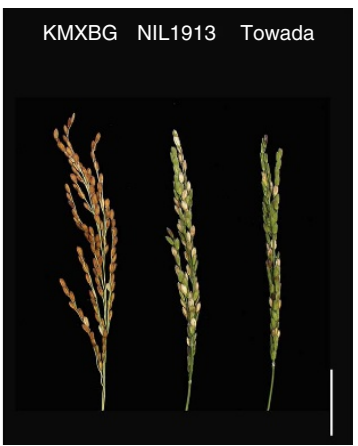

CS-HAA
C

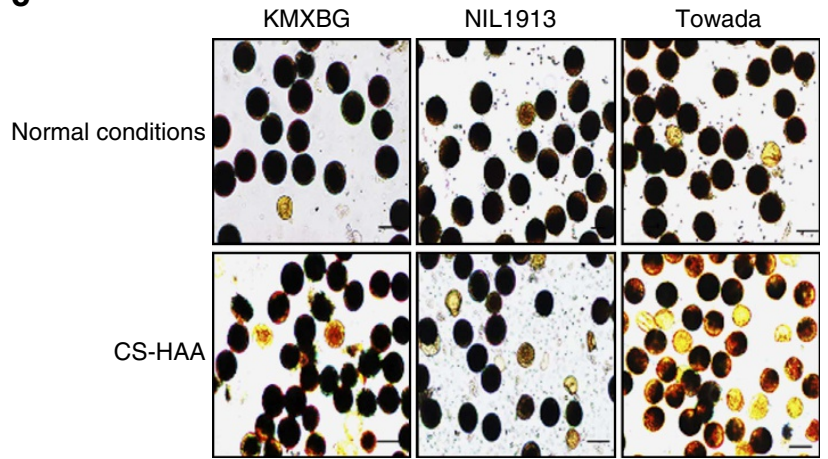

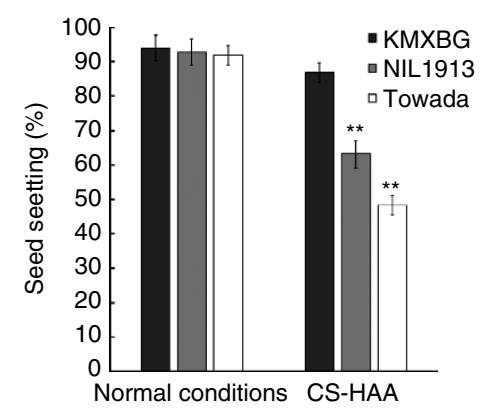

d

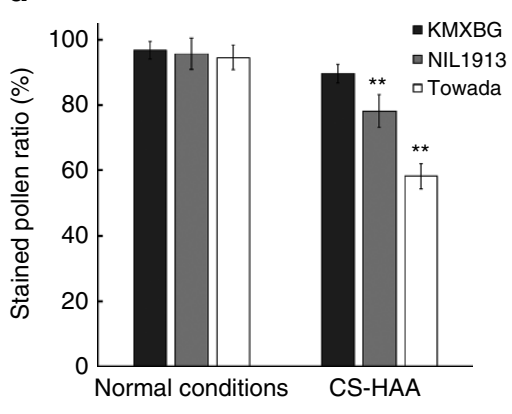

f

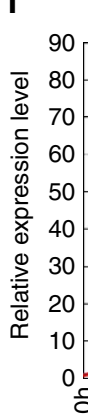

-

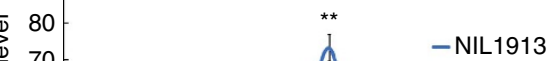

- Towada

e
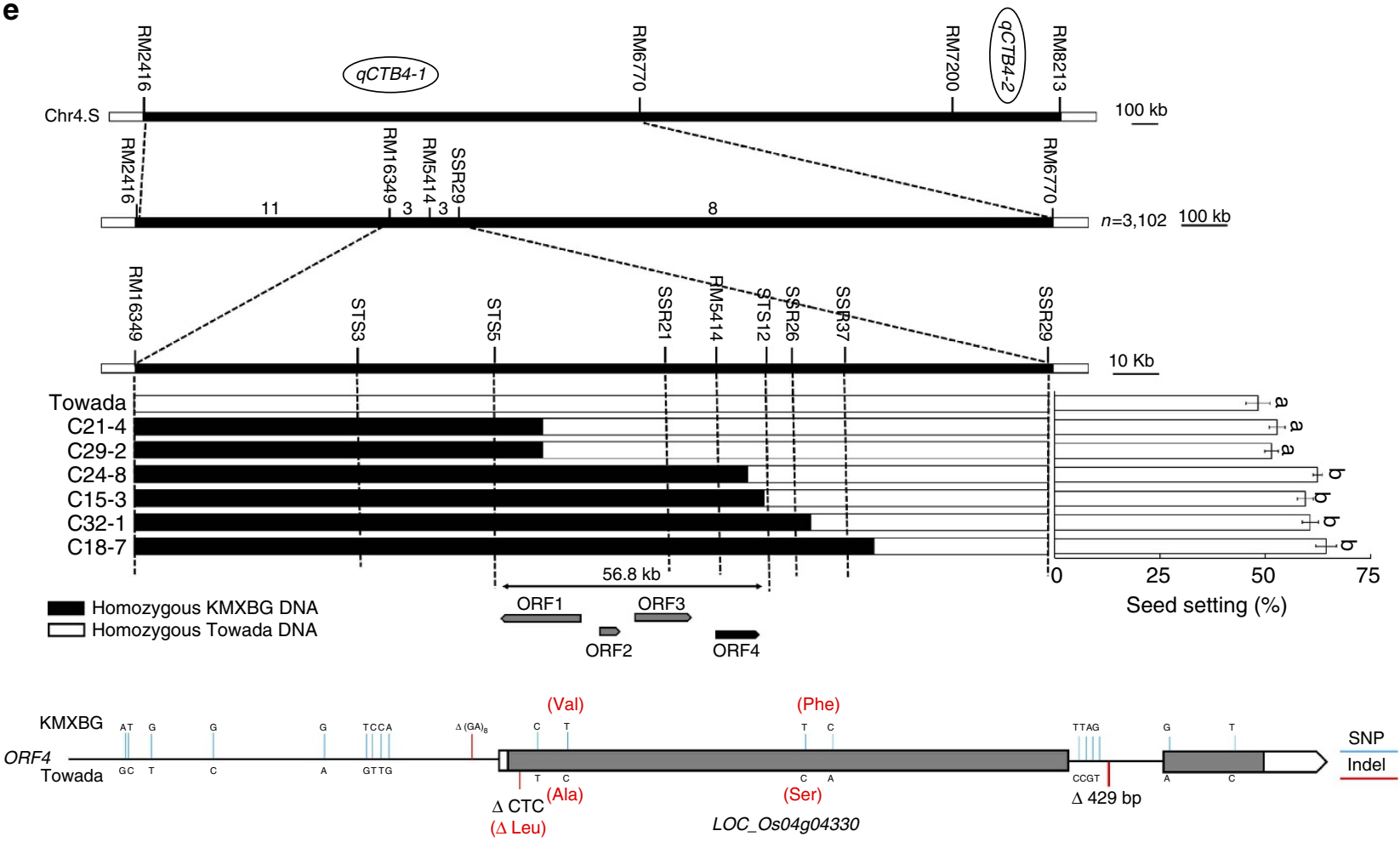

Figure 1 | Characterization of NIL1913 and identification of CTB4a. (a,b) Panicles (a) and seed setting (b) of KMXBG, Towada and NIL1913 planted under normal conditions (Beijing) and cold stress at a higher-altitude area (CS-HAA, Kunming). Data represent means \pm s.d. $(n=15),{ }^{\star \star} P<0.01$, ${ }^{\star} P<0.05$, Student's t-test. Scale bars, $4 \mathrm{~cm}$. (c,d) Pollen fertilities evaluated by $\mathrm{I}_{2}-\mathrm{KI}$ staining $(\mathbf{c})$, and the blue stained pollen grains were counted for calculating pollen fertility for samples under CS-HAA (d). Scale bars, $25 \mu \mathrm{m}$. Data represent means \pm s.d. $(n=10)$. (e) Fine mapping of CTB4a. Left, high resolution mapping. Right, phenotypes of homozygous recombinants. Significance was determined by one-way ANOVA. Schematic of the gene structure and allelic variation in CTB4a between KMXBG and Towada indicated by vertical lines at bottom. The presence of the same lowercase letter above the error bar denotes a non-significant difference between the means ( $P>0.05$, Student's $t$-test). (f) qRT-PCR analysis using total RNA isolated from panicles of NIL1913 and Towada under CS-PT at different time points. Data represent means \pm s.d. $(n=3),{ }^{\star} P<0.01,{ }^{\star} P<0.05$, Student's $t$-test. 
a
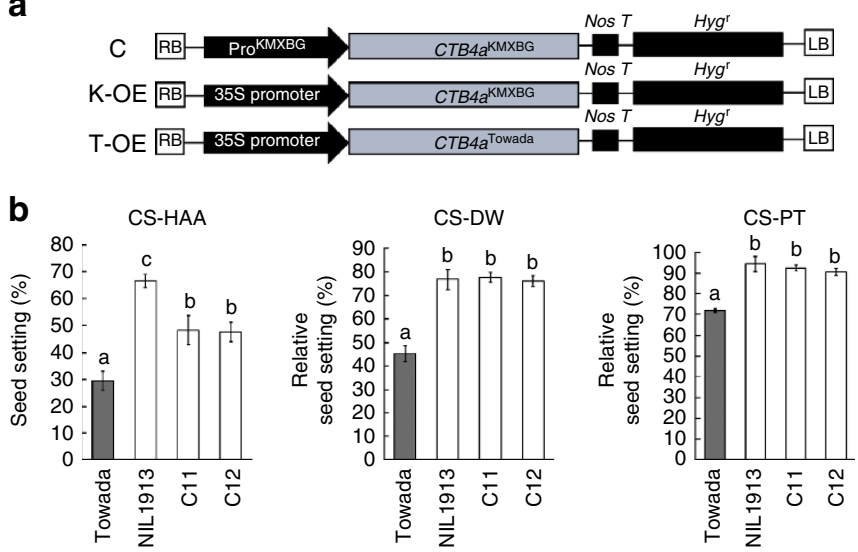

C
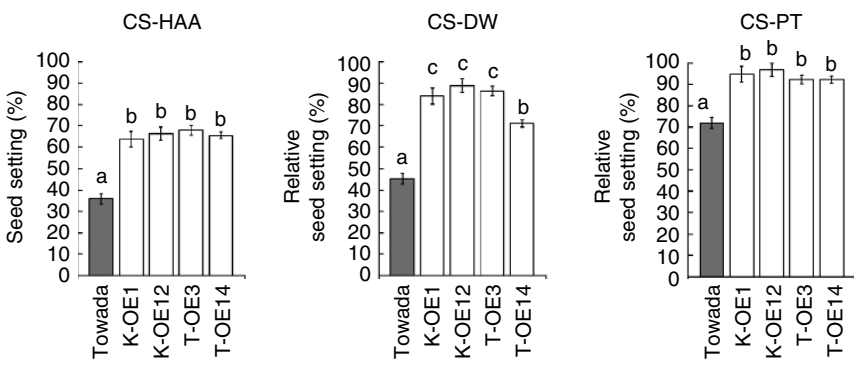

e
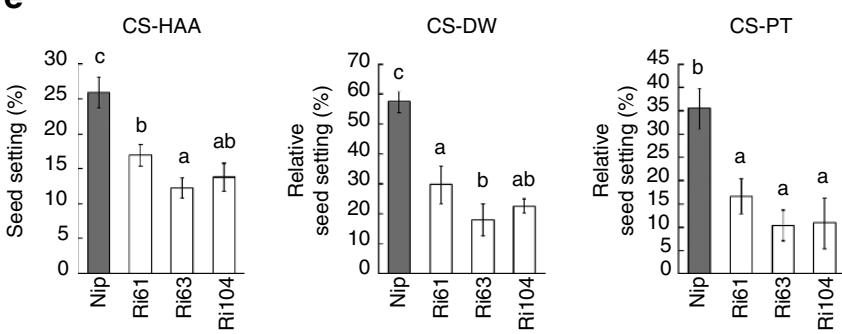

d
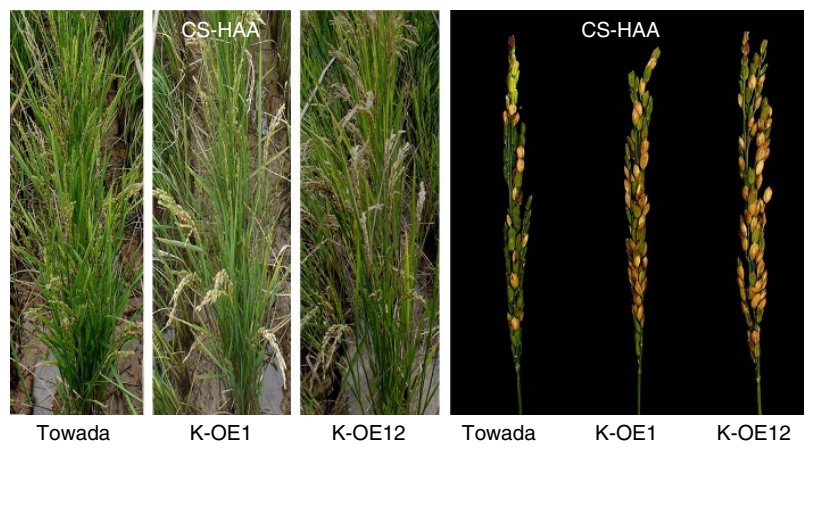

f
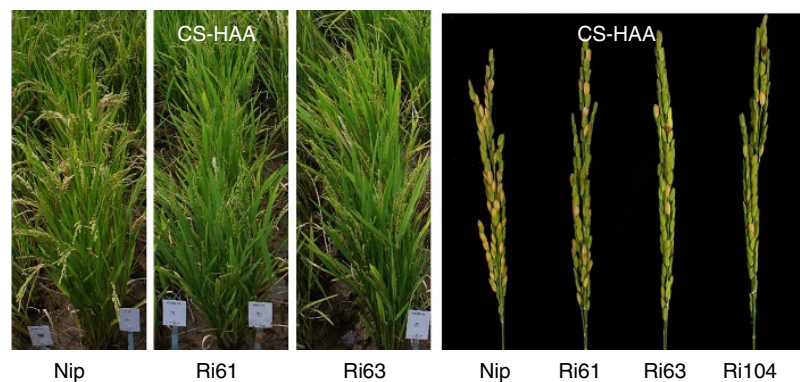

g
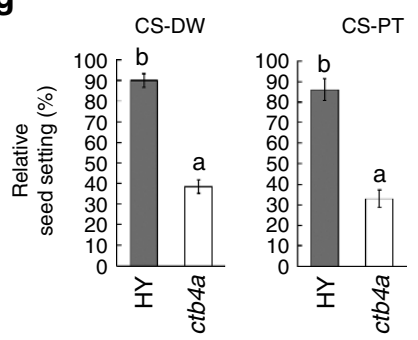

h

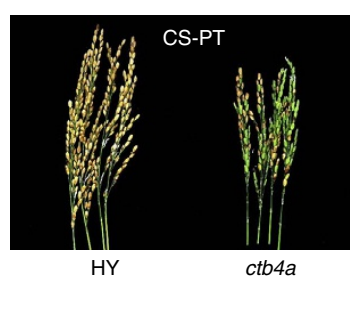

Figure 2 | Functional analysis of CTB4a at the booting stage. (a) Schematic of vectors for transgenic analysis. C, complementation vector; K-OE, CTB4 $a^{\text {KMXBG }}$ overexpression vector; T-OE, CTB4a Towada overexpression vector. (b) Statistical results for seed setting of Towada, NIL1913, C11 and C12 under cold stress. Data represent means \pm s.d. $(n=20)$. (c) Statistical results for seed setting of Towada and overexpression lines under cold stress. Data represent mean \pm s.d. $(n=25)$. (d) Phenotype of plants and panicles of overexpression lines grown under CS-HAA. (e) Statistical results for seed setting of Nip and RNAi lines under cold stress. Data represent means \pm s.d. $(n=15)$. (f) Phenotype of plants and panicles of RNAi lines grown under CS-HAA. (g) Phenotypic analysis of HY and ctb4a grown under CS-PT and CS-DW. Data represent means \pm s.d. $(n=10)$. (h) Panicles of HY and $c t b 4 a$ mutant under CS-PT. The presence of the same lowercase letter above the error bar denotes a non-significant difference between the means $(P>0.05$, Student's $t$-test).

stable and obviously enhanced cold tolerance compared to Towada under CS-DW, CS-PT and CS-HAA conditions over several years (Fig. 2c,d and Supplementary Figs 6b,8). Similarly, T-OE3 and T-OE14 exhibited enhanced cold tolerance with significantly increased seed setting and showed little difference with K-OE1 and K-OE12 (Fig. 2c and Supplementary Fig. 8). Moreover, we assayed the transcript abundance of CTB4a in different overexpression lines and calculated the correlation between CTB4a expression level and seed setting. We found that the cold tolerance of different lines had a high correlation $\left(R^{2}=0.7477\right)$ with CTB4a expression level (Supplementary Fig. 9). These results indicate that the Towada allele of CTB4a is also functional. The different cold sensitivities of KMXBG and Towada are therefore more likely attributed to nucleotide differences in the promoter region.

To further test the function of CTB4a in cold tolerance, an RNA interference (RNAi) vector was constructed and transformed into Nip (as we failed to obtain regenerated plants from calli of KMXBG and NIL1913). Downregulation of CTB4a in Nip resulted in a significant reduction in seed setting in RNAi lines under cold stress (Fig. 2e,f and Supplementary Fig. 6c). In addition, a loss-of-function mutant, $c t b 4 a$, showed significantly reduced seed setting compared to its wild type, Hwayoung (HY), under cold stress (Fig. 2g,h and Supplementary Fig. 10a-c). All of these results suggest a dosage effect of CTB4a on cold tolerance at the booting stage.

When rice plants were exposed to low temperatures at the booting stage, anther injury often occurred and led to failure of microspore or pollen development ${ }^{27}$. We found that the anthers of Towada were more seriously injured with a higher proportion of distorted pollen chambers showing unusual patterns of adhesion (Supplementary Fig. 11a-c) and with reduced pollen fertility compared to those of NIL1913 (Fig. 1c,d) and K-OE lines (Supplementary Fig. 12) under cold stress as evidenced by tissue 
fractionation, although pistil development was unaffected (Supplementary Fig. 11d). On the contrary, the ctb4a mutant showed much lower pollen fertility than HY (Supplementary Fig. 10d,e). When emasculated spikelets of cold stressed Towada and NIL1913 plants were hand-pollinated with pollen from noncold-stressed plants, there was no significant difference in the seed setting (Supplementary Fig. 11e). These results demonstrate that $C T B 4 a$ confers cold tolerance with increased seed setting via its effect on pollen fertility, but with no apparent effect on female fertility.

KMXBG also showed strong cold tolerance at the vegetative growth stage $\mathrm{e}^{25,26}$. Strong GUS signals were detected in buds and young leaf sheaths of pCTB4a KMXBG.:GUS transgenic plants (Supplementary Fig. 13). This information prompted us to further investigate the chilling or cold tolerance at the seedling and germination stages. Survival rates of the overexpression and complementation lines were significantly higher than Towada after chilling stress (Supplementary Fig. 14a,b). On the contrary, the RNAi lines showed decreased chilling tolerance compared to Nip (Supplementary Fig. 14c,d). Moreover, the overexpression lines showed much quicker germination than Towada under cold stress (Supplementary Fig. 15). These results indicate that CTB4a plays a significant role during vegetative growth as well as at the booting stage.

Expression pattern of CTB4a. In order to explore the subcellular localization of CTB4a, we introduced a CTB4a-GFP fusion gene under control of the CaMV35S promoter into Nip. Fluorescent signals of GFP were microscopically detected on the cell surface and in chloroplasts in rice leaf sheath cells (Supplementary Fig. 16a). Moreover, the same signal location of GFP and the cell membrane stain dye Dil were observed in root cells (Supplementary Fig. 16b). In addition, transient expression of CTB4a-GFP in tobacco leaf cells confirmed the subcellular localization of CTB4a-GFP to the cell surface and chloroplasts (Supplementary Fig. 16c,d).

To further examine the expression of CTB $4 a$ in different rice tissues, several experiments were conducted. Quantitative RTPCR analysis showed that CTB4a is widely expressed in root, stem, leaf, sheath and panicle (Supplementary Fig. 17a). Histochemical analysis of pCTB4a $\mathrm{KMXBG}_{:: G U S}$ transgenic plants revealed strong GUS activity in different tissues, especially the anthers (Supplementary Fig. 17b). In situ hybridization indicated that CTB4a was strongly expressed in the tapetum and anther connective vascular bundle (Supplementary Fig. 17c), which was consistent with the effect of $C T B 4 a$ on pollen fertility. This expression pattern of $C T B 4 a$ is consistent with the observed phenotypes in anther tissue.

CTB4a promoter region variation and cold tolerance. To test for an association between CTB4a alleles and cold tolerance at the booting stage in diverse rice germplasm, we evaluated the cold tolerance of 46 japonica and 73 indica cultivars (Supplementary Data 1). As expected, japonica cultivars showed more cold tolerance than indica cultivars (Supplementary Fig. 18). According to the nucleotide polymorphisms identified in the two parents (KMXBG and Towada), we could divide the sequences of the 119 cultivars into nine haplotypes, among them Hap1, Hap7, Hap8 and Hap9 present only in the japonica (sub-J), Hap3, Hap4 and Hap6 only in indica (sub-I), and Hap2 and Hap5 both in sub-J and sub-I subpopulations. Both parent haplotypes Hap1 (HapKMXBG) and Hap9 (Hap-Towada) were present in sub-J (Fig. 3a). Furthermore, phylogenetic analysis showed that the nine haplotypes could be divided into three groups with Hap1 in Group A, Hap2 to Hap7 in Group B, and Hap8 and Hap 9 in
Group C (Fig. 3b). Accessions in Group A (only Hap1) showed obviously stronger cold tolerance than Group B and C (Fig. 3c). Also, 18 wild rice accessions were sequenced and although we found similar diversity to that in cultivated rice accessions, no haplotypes identical to Hap-KMXBG or Hap-Towada were found (Fig. 3a). These results suggest that the cold tolerant haplotype of CTB $4 a$ may have originated in japonica and may be present only in japonica (although it is possible that it may be present in accessions not studied here).

All accessions in Hap-KMXBG with five consensus SNPs (SNP-2536, SNP-2511, SNP-1930, SNP-780 and SNP2063) exhibited apparently higher cold tolerance than other haplotypes (Fig. 3a-c). SNP-2536 and SNP-2511 carried cis element (gibberellin response element ${ }^{28}$ and anaerobic induced regulatory element ${ }^{29}$ ) changes according to PLACE (Plant cisacting regulatory DNA elements) analysis. We conducted transient assays of the site-directed mutated promoter fragments of CTB4a in Arabidopsis protoplasts to test the effects of the four SNPs in the promoter region (Fig. 3d). The relative activities of the promoter fragments with SNP-2536, SNP2511 and SNP-1930 were significantly reduced (Fig. 3d). These results indicate that Hap-KMXBG (Hap1) containing all five consensus SNPs in the promoter region represents the coldtolerant haplotype and that SNP-2536, SNP-2511 and SNP-1930 are functional SNPs (FNPs) accounting for the expression level differences between the CTB4a alleles.

Low-temperature acclimation of Tej-Hap-KMXBG. In regard to geographic distribution of the nine haplotypes, we found that Hap-KMXBG (Hap1) mostly occurred in northeastern China (N: $40-50^{\circ}$; Supplementary Fig. 19). To examine the detailed geographic distribution of the alleles, we classified the 119 accessions as two kinds of haplotypes, namely Hap-KMXBG and Hap-others. Twenty seven cultivars with Hap-KMXBG were from the northern areas of China, Japan, Korea and Western Europe or from the high-altitude southwestern zone of China (YunnanGuizhou plateau with an average elevation of 2,000 m). Hapothers were mainly distributed in regions with higher temperatures, such as southern China and southeastern Asia (Fig. 4a). Moreover, analysis of phylogenetic relationship of CTB $4 a$ based on 196 SNPs in 459 accessions including 11 wild rice (O. rufipogon) lines and 448 varieties from the world rice core collection (Supplementary Data 1) showed that CTB4a was quite diverse across the range of indica, japonica, Aro and Aus accessions (Fig. 4b). We identified 25 CTB4a haplotypes among 459 accessions, and only three haplotypes contained the same FNPs as Hap-KMXBG, but none was wild rice (Fig. 4c). These results imply that Hap-KMXBG does not directly originate from O. rufipogon during japonica domestication.

The three haplotypes containing the same FNPs as HapKMXBG specifically existed in the temperate japonica (Tej) group suggesting that this unique haplotype, named as Tej-HapKMXBG, was selected during evolution. We analysed the nucleotide diversity of CTB4a among indica, Tej, tropic japonica (Trj) and O. rufipogon to determine whether selection had acted on CTB4a. On average, the nucleotide diversity of CTB4a in Tej was higher $(\pi=0.00351)$ than in indica $(\pi=0.00223)$ or $\operatorname{Trj}$ $(\pi=0.00271)$. When Tej was further divided into Tej-HapKMXBG and Tej-Hap-Towada, the $\pi$ value in Tej-Hap-KMXBG $(\pi=0.00018)$ was much lower than that in Tej-Hap-Towada $(\pi=0.00266)$, indica, Trj and O. rufipogon $(\pi=0.00413$; Fig. $4 \mathrm{~d}$, Supplementary Fig. 20 and Supplementary Table 2). Such decreased diversity could be a result of positive selection or other demographic situation, such as a bottleneck effect ${ }^{30,31}$. In an attempt to discriminate the two effects, we calculated the 
a

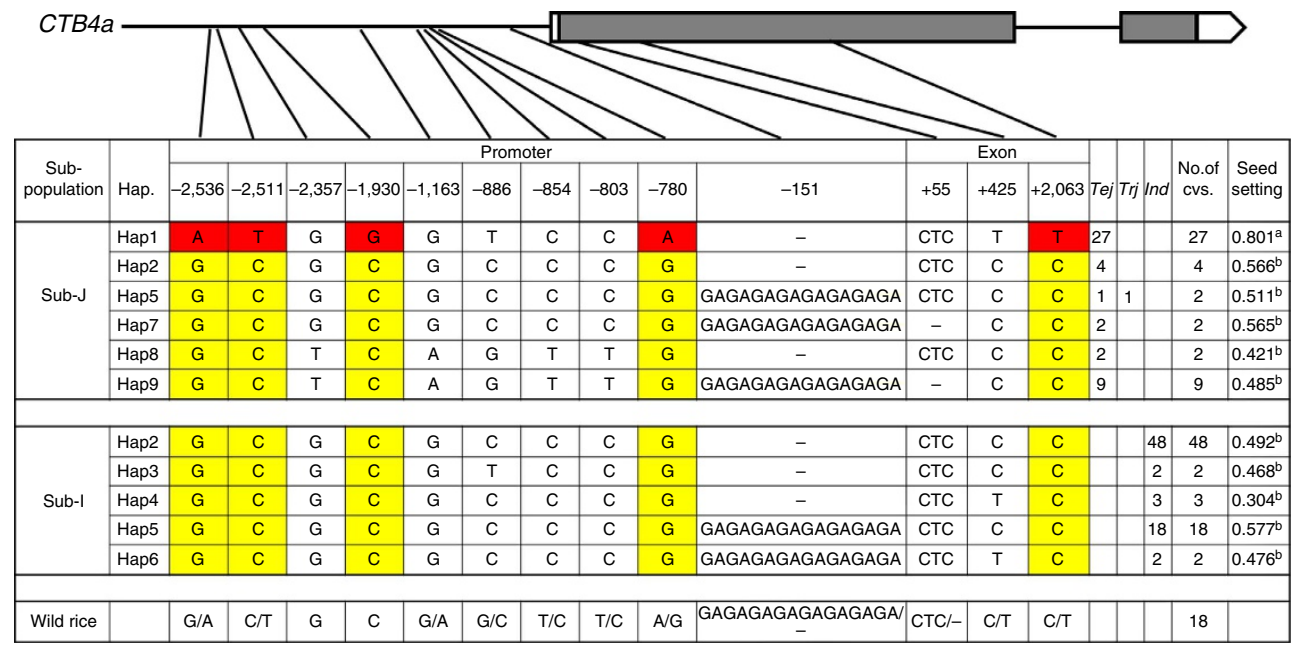

b

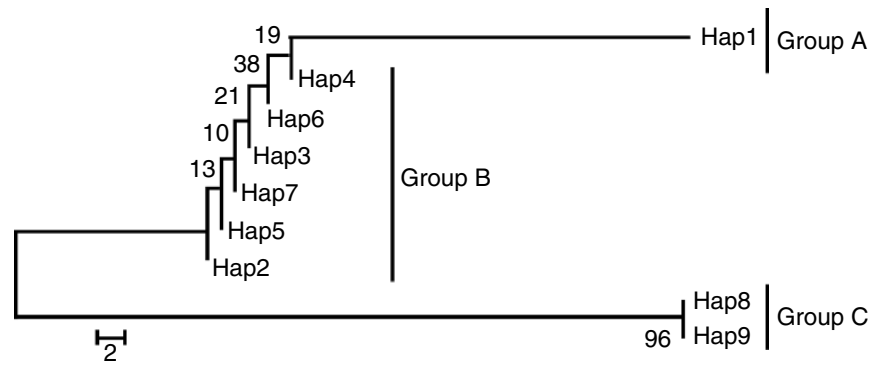

C

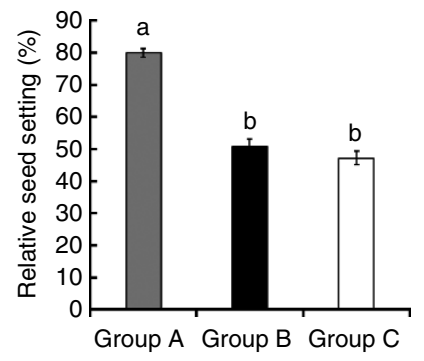

d

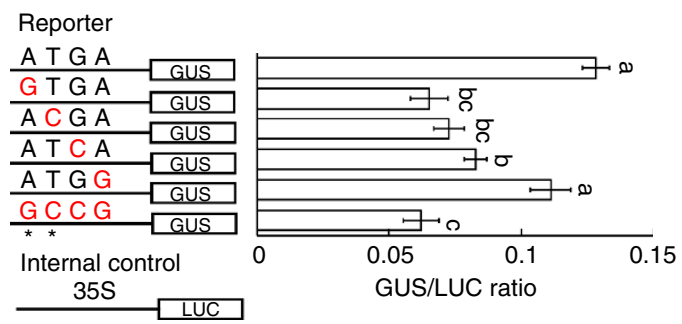

Figure 3 | Haplotype analysis of CTB4a. (a) Haplotype analysis of the CTB4a gene region from 119 rice cultivars and 18 O. rufipogon accessions. Ind, indica population; Sub-J, japonica population; Sub-I, indica population; Tej, temperate japonica population; Trj, tropical japonica population. Data represent means $(n=5)$. (b) Phylogenetic tree of the nine haplotypes divided into Groups A (Hap1), B (Hap2- Hap7) and C (Hap8- Hap9). The scale bar indicates average number of substitutions per site for different haplotypes. (c) Relative seed setting of the three Groups. Data represent means \pm s.d. (d) Transient expression assay of promoter activity in Arabidopsis protoplasts. Left, constructs with site-directed mutations at the four SNPs in the promoter region. Two cis-elements changed at SNP2536 and SNP2511 indicated with '*'. Right, relative GUS/LUC values. The presence of the same lowercase letter above the error bar denotes a non-significant difference between the means $(P>0.05$, Student's $t$-test).

nucleotide diversity of $20 \mathrm{~kb}$ flanking regions of CTB4a. The average nucleotide diversity of the CTB4a flanking regions in Tej-Hap-KMXBG was significantly lower than that among others $(P<0.001$, student's $t$-test; Supplementary Table 3$)$. As positive selection will result in range of genomic changes, resembling to our observation on the polymorphism pattern of Tej-HapKMXBG, the decrease of nucleotide diversity of CTB4a in Tej may be largely caused by positive selection. Also significant Tajima's $D$ (ref. 32) values were observed in Tej-Hap-KMXBG cultivars (Supplementary Table 2), which were consistent with selection at CTB4a locus. To further determine whether the reduction of nucleotide diversity in Tej-Hap-KMXBG was caused by artificial selection, we used Maximum likelihood HudsonKreitman-Aguade (MLHKA) tests on CTB4a sequences for six groups and seven neutral genes ${ }^{33}$ as a control. A significant value was observed for Tej-Hap-KMXBG $(P=3.132 \mathrm{E}-05$, student's $t$ test) indicating that a strong artificial selection occurred on the CTB4a locus during japonica domestication (Supplementary Table 2). These results collectively indicate that Tej-HapKMXBG of CTB4a representing a new allele generated from natural variation in cooler environment is retained by artificial selection for low-temperature acclimation in rice.

CTB4a interaction with AtpB. To explore the potential mechanism by which CTB4a influences cold tolerance, we screened for interacting proteins using truncated CTB4a with the kinase domain $\left(\mathrm{CTB} 4 \mathrm{a}^{\mathrm{KD}}\right)$ as bait and identified three candidates, 
a

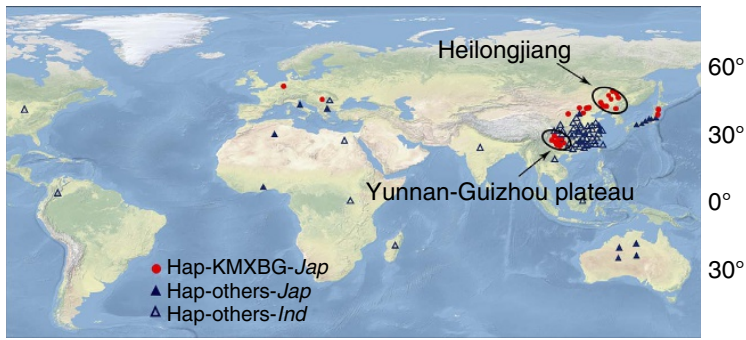

b

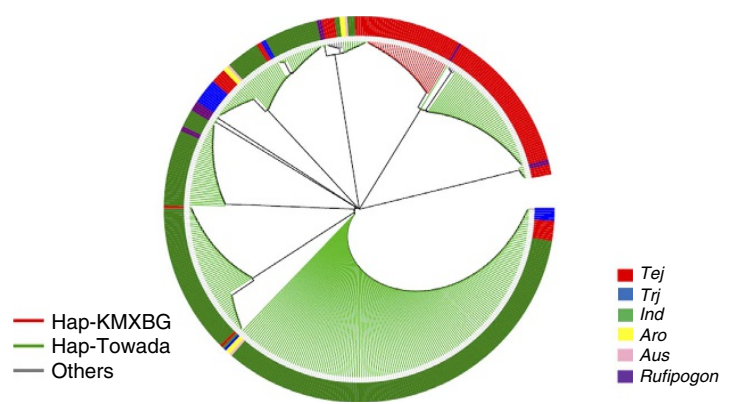

C

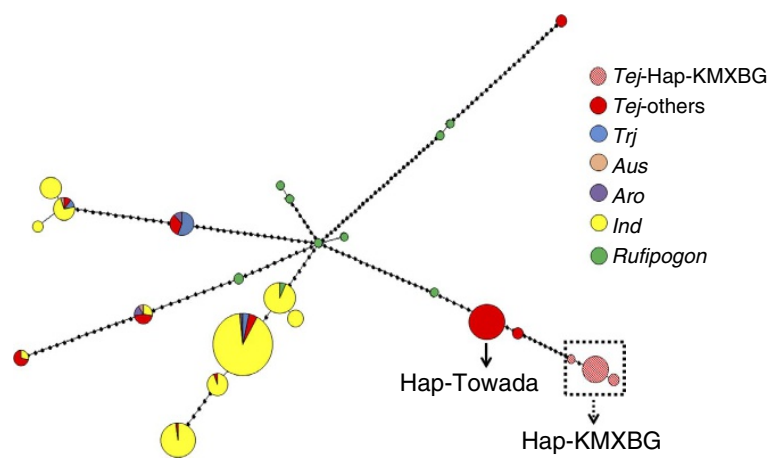

d

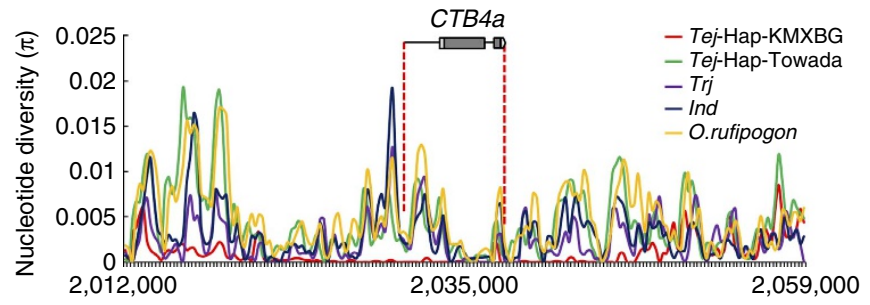

Figure 4 | The geographic and phylogenetic origins of CTB4a.

(a) Geographic distributions among 119 accessions. The japonica HapKMXBG members are indicated by red circles. Other japonica and indica accessions are indicated by solid blue or hollow triangles, respectively. (b) Phylogram of CTB4a generated from 459 diverse rice accessions including the Tej, Trj, Ind, Aro, Aus and O. rufipogon showing divergence between the Hap-KMXBG and Hap-Towada. (c) Haplotype network of CTB4a. Circle size is proportional to the number of samples for a given haplotype. Black spots represent unobserved, but inferred haplotypes. Lines between haplotypes represent mutational steps between alleles. The black square with dotted arrow encloses rice lines with the CTB4a Hap-KMXBG type SNPs and the solid arrow indicates the CTB4a SNPs of Hap-Towada type. (d) Nucleotide diversity of CTB4a in cultivar populations and the wild rice group. The $\mathrm{X}$-axis denotes the position of CTB4a and the $\mathrm{Y}$-axis indicates average $\pi$ values. two of which were beta subunits of ATP synthases and one of which was named AtpB (Fig. 5a and Supplementary Table 4). Interaction between $\mathrm{CTB} 4 \mathrm{a}^{\mathrm{KD}}$ and AtpB in vitro was confirmed by a GST pull down assay (Fig. 5b) and in vivo by a co-immunoprecipitation (Fig. 5c). Bimolecular fluorescence complementation assays performed in Nicotiana benthamiana showed that both the CTB4a $\mathrm{a}^{\mathrm{KMXBG}}-\mathrm{YFP}^{\mathrm{N}}$ and CTB4a $\mathrm{a}^{\text {Towada }}$ $\mathrm{YFP}^{\mathrm{N}}$ could interact with AtpB-YFPC at chloroplasts, but not YFPC $^{C}$ alone (Fig. 5d). Next, we found that ATP synthase activity and ATP content as well as photosynthetic rate, were significantly higher in leaves of NIL1913 and the overexpression lines than in Towada (Fig. 6a), but were significantly lower in $c t b 4 a$ compared to $\mathrm{HY}$ at the booting stage under cold stress (Fig. 6b). Also higher ATP content were observed in panicles of NIL1913 and the overexpression lines than in Towada (Fig. 6c), and reduced ATP content in panicles of $c t b 4 a$ compared to HY under cold stress (Fig. 6d). Moreover, exogenous application of ATP partly rescued the cold sensitivity of Towada (Fig. 6e). ATP supplies energy for plant growth and metabolism. We found that NIL1913 and overexpression lines showed higher yield with increased filled grain number per-panicle and thousand-kernel weight than Towada under cold stress (Fig. $6 \mathrm{f}$ and Supplementary Fig. 21).

To further investigate the function of $A t p B$ for cold tolerance, we obtained $A t p B$ overexpression lines and found that $A t p B-\mathrm{OE} 3$ and $A t p B-O E 24$ showed significantly increased seed setting than that of Nip under cold stress at the booting stage (Fig. $6 \mathrm{~g}$ and Supplementary Fig. 22). These results collectively indicate that CTB4a can interact with AtpB. It is possible that this interaction mediates ATP synthesis to ensure an adequate energy supply to enhance seed setting and increase yield in cold rice growing areas.

\section{Discussion}

In general, seed setting of plants stressed by natural low temperatures or artificially controlled low temperatures is used to evaluate the cold tolerance at the booting stage in rice ${ }^{34}$. However, the differences in rice growth period and the limitations of equipment used for cold evaluation have restricted the accuracy which with cold tolerance at the booting stage in rice can be assessed. Compared with other agronomic traits, the evaluation of cold tolerance of rice, especially at the booting stage, is more difficult. For example, a total of 16 years were spent for fine mapping and cloning of Ctb1 (ref. 24). Based on these reasons, we constructed a series of cold-tolerant near-isogenic lines using KMXBG, a strong cold-tolerant native Yunnan cultivar, as the donor parent and Towada as the recipient parent to exclude the influence of the different heading date and genetic backgrounds, which laid the foundation for the isolation of genes for cold tolerance at the booting stage. In addition, we established three methods to evaluate the cold tolerance of the plant materials. High-elevation environments where lowtemperature stress occurs provides a reliable location to study a large number of genotypes, which is necessary for the application of map-based cloning of the QTLs for cold tolerance.

Low temperatures lead to a series of changes at the molecular and cellular levels as well as physiological and biochemical levels in plant cells. Plants need energy to reinforce resistance to cold stress, among which ATP is one of the important energy sources $^{35-38}$. Our results demonstrated that upregulation of CTB $4 a$ could increase ATP synthase activity and ATP content in NIL1913 and overexpression lines under cold stress at the booting stage (Fig. 6a-d). Higher pollen fertility was observed in NIL1913 and $C T B 4 a$ overexpression lines than Towada under cold stress (Fig. 1c,d and Supplementary Fig. 12). Moreover, the application of exogenous ATP increased seed setting of Towada under cold stress (Fig. 6e). We therefore suggest that the low expression of 
a

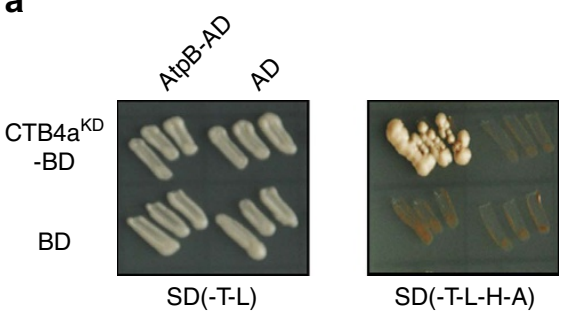

c

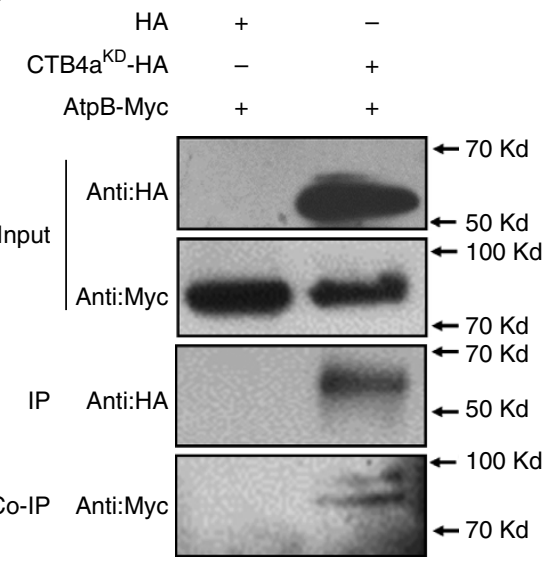

b

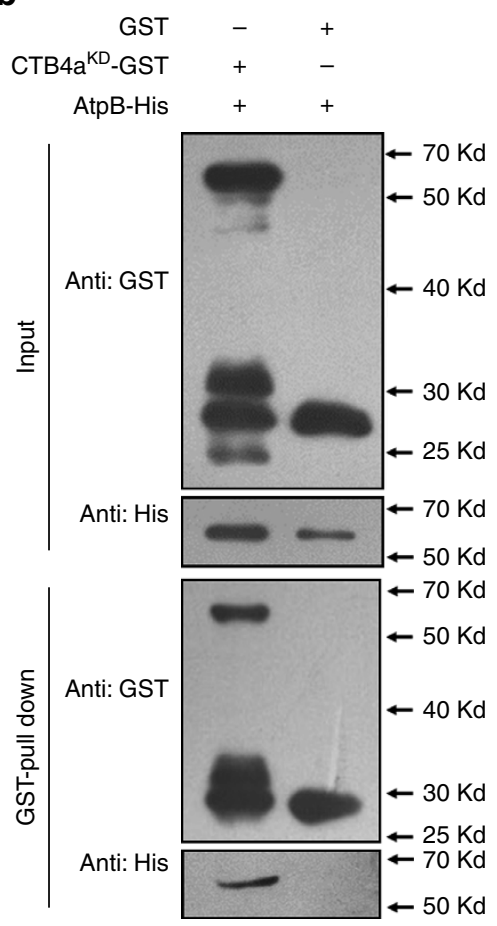

d

YFP

\begin{abstract}
Bright
\end{abstract}
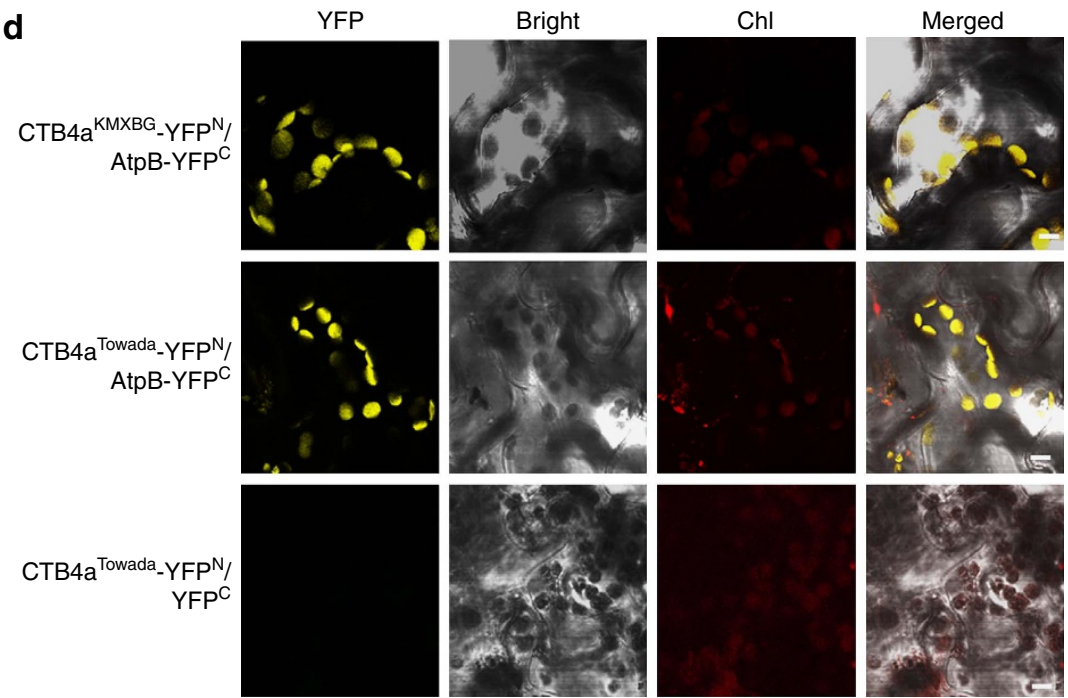

Figure 5 | CTB4a can interact with AtpB. (a) Interaction assay of CTB4a KD and AtpB in yeast (strain AH109). Interaction was determined by a growth assay on medium lacking Trp-Leu-His-Ade. (b) GST-pull down assay confirming the interaction between CTB4aKD and AtpB in vitro. Purified CTB4a KD -GST and AtpB-His fusion protein from E.coli BL21 were pull-downed by GST beads and blots were probed with anti-GST or anti-His. (c) Interaction assay of CTB4a ${ }^{K D}$ and AtpB in vivo. Co-expressed CTB4aKD-HA or HA and AtpB-Myc in tobacco leaves were immunoprecipitated by anti-HA antibody and blots were probed with anti-Myc or anti-HA. (d) Bimolecular fluorescence complementation assay. Chl, chloroplast; YFP, yellow fluorescent protein. Scale bar, $5 \mu \mathrm{m}$.

CTB4a and reduced ATP content in Towada lead to less energy for cold tolerance and reduced pollen fertility and decreased seed setting. It was previously reported that a shortage of ATP can reduce grain productivity ${ }^{39,40}$. We finally found that NIL1913 and CTB4a overexpression lines exhibited improved grain yield under CS-HAA (Fig. 6f).

Phosphorylation can affect ATP synthase activity or assembly, and this modification often occurs in the $\beta$ subunit which plays catalytic function ${ }^{41}$. The $\beta$ subunit of chloroplast ATP synthase can be phosphorylated by kinases and this has been reported in a variety of plants ${ }^{42,43}$. However, the phosphorylation of ATP synthase has not been well documented in rice. We found that
CTB4a exhibited autophosphorylation activity but could not phosphorylate AtpB in vitro (Supplementary Fig. 23). Many plant RLKs undergo a signal perception, self-phosphorylation, dimerization and trans-phosphorylation processes to activate basal kinase function ${ }^{44}$. Our data indicated that CTB4a can interact with AtpB, and that upregulation of CTB4a increased ATP synthesis. Overexpression of $A t p B$ also increased cold tolerance of rice at the booting stage (Figs 5 and 6a,g). It is possible that AtpB may be phosphorylated by CTB4a in vivo. In addition, it could also be possible that CTB4a interacts with AtpB to enhance the phosphorylation of AtpB by other kinases, thus affecting ATP synthase activity or may regulate ATP production 
a

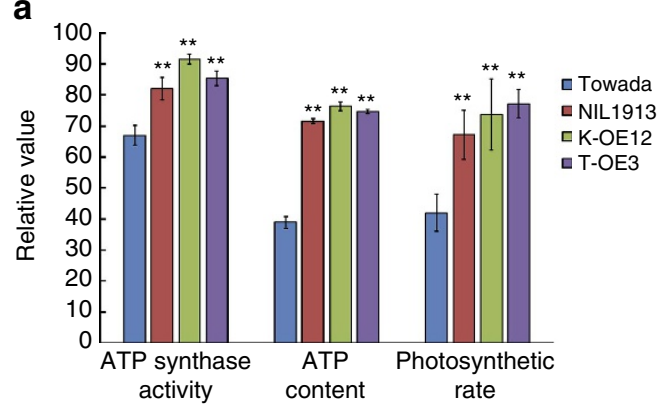

b

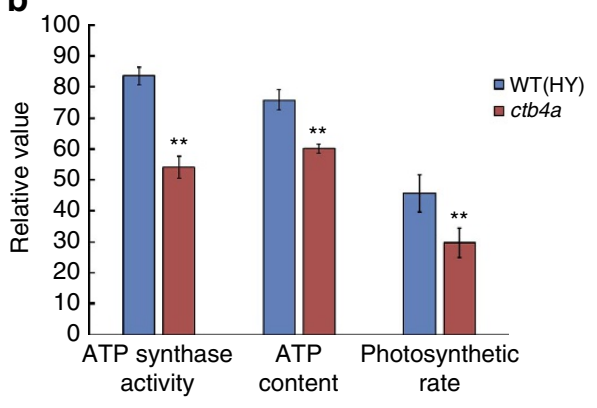

C

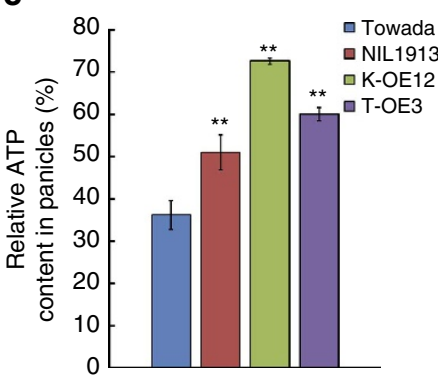

d

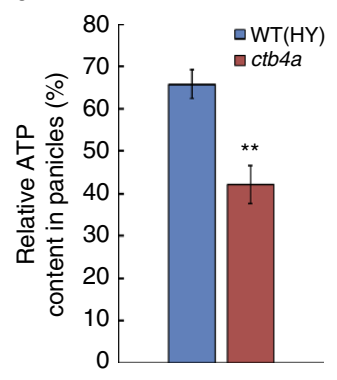

e

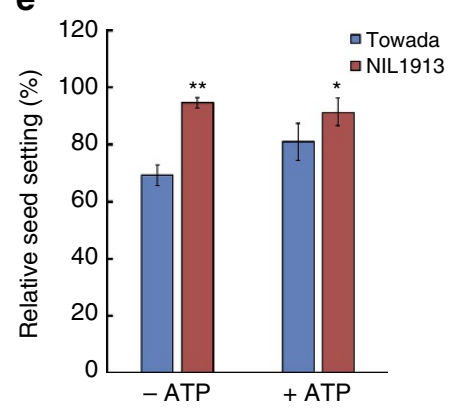

f
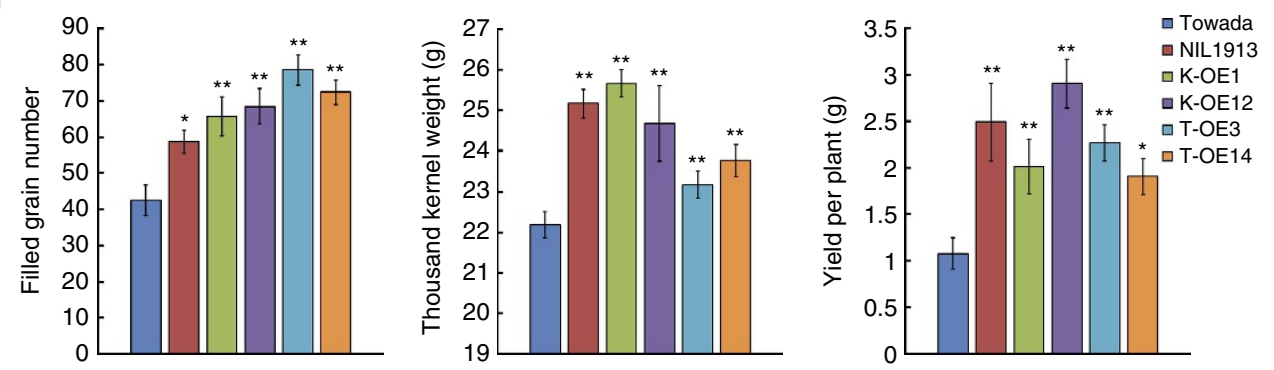

g

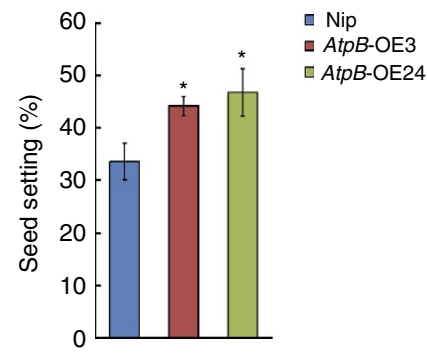

Figure 6 | CTB4a mediates ATP supply and improves rice yield. (a) ATP synthase activity, ATP content and photosynthetic rate in leaves of Towada, NIL1913 and overexpression lines under CS-PT for 3 days. (b) ATP synthase activity, ATP content and photosynthetic rate in leaves of HY and ctb4a mutant under CS-PT for 3 days. (c,d) ATP content in panicles of overexpression lines (c) and ctb4a mutant (d). (e) Relative seed setting of Towada and NIL1913. Exogenously applied ATP ( $5 \mathrm{mM})$ rescued the cold sensitivity of Towada. Data represent means \pm s.d. $(n=15)$. (f) Filled grain number per-panicle, thousand-kernel weight and yield per-plant in Towada, NIL1913 and transgenic plants under CS-HAA. Data represent means \pm s.d. $(n=30)$. $(\mathbf{g})$ Statistical results for seed setting of Nip and AtpB overexpression lines under CS-PT. Data represent means \pm s.d. $(n=5)$. All $P$ values were calculated by Student's t-test. ${ }^{\star \star} P<0.01,{ }^{\star} P<0.05$.

in a different manner, such as via the assembly of the ATP synthase complex under cold stress conditions. These hypotheses remain to be verified in the further studies.

The progress in cloning CTB4a began with a tightly linked QTL in the gene region (Fig. 1e and Supplementary Fig. 3a). The mining and pyramiding of important QTLs is an effective method to produce varieties with high yield, good quality and stress tolerance. CTB4a is a conserved LRR-RLK in plants (Supplementary Fig. 4b), especially in gramineous plant where protein identity exceeded more than 66\% (Supplementary
Fig. 4c). Use of CTB4a and its homologous genes could potentially accelerate production and use of cold tolerant varieties in crop plants. During crop domestication, the genetic modification of a wild species could alter phenotypic characteristics to meet human demands, in which natural selection plays an important role to maintain the genetic diversity of cultivars ${ }^{45,46}$. We found that Tej-Hap-KMXBG of CTB4a had been retained in landraces and recently improved varieties, such as DAOHUAXIANG, KENDAO 12, DONGNONG 428, LIJIANGXINTUANHEIGU, YUNJING 23 and YUNDAO 1, of 
temperate japonica in northeastern China and the Plateau of Lijiang (altitude 2, $384 \mathrm{~m}$ ), Yunnan (Supplementary Data 1). With wide spread planting of varieties with Tej-Hap-KMXBG of $C T B 4 a$, Heilongjiang province $\left(\mathrm{N}: 43-53^{\circ}\right)$, referred to as the 'cold rice growing zone' (Supplementary Fig. 24), has become the largest rice area in China at 4.3 million hectares per year. It is expected that Tej-Hap-KMXBG of CTB4a will allow rice production to expand in the currently cooler areas.

To our knowledge, CTB4a is the first LRR-RLK gene conferring cold tolerance at the booting stage isolated by forward genetics in rice. More importantly, NIL1913 and CTB4a overexpression lines showed stable cold tolerance not only in the greenhouse but also in field conditions over several years (Fig. 2 and Supplementary Figs 2,8). On the basis of the alleles and FNPs identified in this work, we suggest that CTB4a has great potential for improving rice cold tolerance at the booting stage via molecular breeding techniques. New cultivars designed on the basis of our study could test whether CTB4a can confer cold tolerance in other crops in the future.

In summary, this work has uncovered a novel gene CTB4a controlling cold tolerance at the booting stage in rice. Our findings suggest that the natural variation in the promoter region of CTB4a may have enhanced rice adaptation to cold habitats during domestication. We propose that CTB4a confers resistance to cold stress by mediating ATP supply to reinforce pollen fertility at low temperature. This gene discovery could be of potential significance for understanding crop domestication and future molecular breeding in crops.

\footnotetext{
Methods

Plant materials. In previous study, we developed a set of cold-tolerant NILs by backcrossing a cold-tolerant landrace, $\mathrm{KMXBG}$ (O. sativa ssp. japonica $\mathrm{cv}$ ), as donor to a cold-sensitive variety, Towada (O. sativa ssp. japonica cv). NIL1913 containing QTL qCTB4-1 was constructed by six-times backcrossing the KMXBG $\times$ Towada hybrid to Towada to generate a $\mathrm{BC}_{6} \mathrm{~F}_{2}$ population. Towada and Nip were used for transformation. CTB $4 a^{\mathrm{KMXBG}}$ overexpression transgenic lines, $C T B 4 a^{\text {Towada }}$ overexpression transgenic lines and complementation lines in Towada background, as well as RNAi lines, $A t p B$ overexpression lines in Nip background were used to evaluate cold tolerance. The $c t b 4 a$, T-DNA insertion mutant in HY (O. sativa ssp. japonica cv.) background, was purchased from the Korean Rice Mutant Center ${ }^{47}$
}

Evaluation of cold tolerance. Three methods were adopted to evaluate cold tolerance at the booting stage. The mapping population was planted under CS-HAA conditions in the summers of 2007, 2008 and 2009. About 30-day-old seedlings were transplanted $(L \times W=12.5 \mathrm{~cm} \times 25 \mathrm{~cm})$ in the field. Cold tolerance of the $\mathrm{F}_{2}$ population was measured by spikelet fertility of the main panicles at seed ripeness, and $\mathrm{F}_{3}$ families were evaluated as average spikelet fertilities of the main panicles of 15 plants per line. $\mathrm{F}_{4}$ families from homozygous recombinant individuals were evaluated as average spikelet fertilities of the main panicles from 10 plants in each line.

The phenotypes of transgenic lines and control plants were evaluated under CSDW and CS-PT in Beijing, and CS-HAA in Kunming as mentioned above. For CSDW, the main panicles at booting were labelled and then transferred to deep cold water $\left(16-18^{\circ} \mathrm{C}\right)$. After one week, the plants were transplanted into the field. The spikelet fertilities of labelled panicles were investigated for evaluating cold tolerance. A similar method to CS-DW was adopted for CS-PT, except that the plants were grown in a phytotron $\left(16-17^{\circ} \mathrm{C}\right)$.

For evaluating cold tolerance at the seedling stage, surface-sterilized seeds were first soaked in water at $28-30^{\circ} \mathrm{C}$ for 2 days and germinated seedlings with similar vigour were planted in pots containing a mixture of soil and vermiculite, and grown in a growth chamber supplied with Hoagland's solution. Four-week-old seedlings were treated with low temperature $\left(4-5^{\circ} \mathrm{C}\right)$ for 7 days and then returned to normal conditions for 10 days. Phenotypes were evaluated by survival rates. At least five pots of 20 plants were assessed for each line.

Germination tests were conducted as described by Fujino at al. ${ }^{19}$ with minor changes. After breaking dormancy, 50 seeds were placed on a filter paper in a $9 \mathrm{~cm}$ Petri dish covered with distilled water. The dishes were placed in an incubator at $16^{\circ} \mathrm{C}$. The number of the germinated seedlings was recorded.

Map-based cloning of CTB4a. High-resolution mapping was conducted using $\mathrm{BC}_{6} \mathrm{~F}_{2}$ populations derived from the cross between NIL1913 and Towada with Towada as the recurrent parent. Two $F_{2}$ populations were used for fine mapping.
One $\mathrm{F}_{2}$ population consisting of 179 individuals was grown at the experimental farm, Yunnan Academy of Agricultural Sciences, Kunming, in the summer of 2007. Another $\mathrm{F}_{2}$ population containing 3,102 individuals derived from the same cross was grown at the China Agricultural University Experiment Station at Sanya $\left(19^{\circ} \mathrm{N}, 109^{\circ} \mathrm{E}\right)$, Hainan province, in the winter of 2007. All $\mathrm{F}_{3}$ families come from the first $\mathrm{F}_{2}$ population, and $\mathrm{F}_{3}$ families from 46 recombinant individuals identified in the second $F_{2}$ population were grown at Kunming in the summer of 2008 for evaluation of cold tolerance. Gene annotation was conducted in RGAP (http://rice.plantbiology.msu.edu/). The primer sequences for genotyping are provided in Supplementary Data 2.

Vector construction and genetic transformation. For construction of a complementation plasmid, a $7.8 \mathrm{~Kb}$ DNA fragment containing the $3.2 \mathrm{~kb}$ promoter, the full $4.1 \mathrm{~kb}$ exons and intron region and a $0.5 \mathrm{~Kb} 3^{\prime}$-UTR of CTB $4 a$ was amplified from KMXBG, digested with PmeI and $A s c I$, and cloned into binary vector pMDC83 (ref. 48). For construction of an overexpression plasmid, the full coding sequences were amplified from the cDNA of KMXBG and Towada, digested with AscI and PacI, and cloned into binary vector pMDC32 (ref. 48), respectively. To construct an RNAi plasmid, the unique fragment was amplified from Nip, digested with SacI and SpeI, and cloned into pTCK303 vector ${ }^{49}$ to generate the forward insertion. Further dsRNAi fragments obtained by digestion with BamHI and KpnI were cloned into the same vector to generate the reverse insertion. For construction of GUS plasmid, $3 \mathrm{~kb}$ DNA fragments containing the CTB4a promoters from KMXBG and Towada were amplified, digested with PmeI and AscI, and inserted into the pMDC162 vector ${ }^{48}$, respectively. For construction of the GFP plasmid, the ORF of CTB4a without stop codon was amplified from the cDNA of KMXBG, digested with SpeI and $A s c \mathrm{I}$, and inserted into the pMDC83 vector ${ }^{48}$. All fragments were amplified by the high fidelity PCR enzyme KOD-FX (TOYOBO, KFX-101). Primer sequences for vector constructions are provided in Supplementary Data 2.

All plasmids confirmed by sequence were introduced into Agrobacterium tumefaciens strain EHA105 and transferred into recipient materials by the Agrobacterium-mediated method ${ }^{50}$.

Expression pattern analysis. For cold-induced expression analysis of CTB4a at the booting stage, NIL1913 and Towada were stressed under CS-PT, and the panicles and leaves were sampled at different time points for RNA extraction. For cold-induced expression analysis of CTB4a at the seedlings stage, leaves of NIL1913 and Towada were sampled from $4-5{ }^{\circ} \mathrm{C}$ conditions at different time points for RNA extraction. For tissue expression pattern analysis, different tissues of KMXBG and Towada were sampled for RNA extraction and quantitative RT-PCR. Total RNA was extracted from different plant tissues using RNAiso Plus (Takara, D9108B). Each experiment was performed with three biological samples and each with three technical replications. OsActin1 was used as a reference. The PCR primer sequences are given in Supplementary Data 2. $\mathrm{T}_{3}$ homozygous transgenic plants containing the pCTB4a ${ }^{\mathrm{KMXBG}}$ ::GUS vector were used for GUS histochemical staining.

Subcellular localization. Leaf sheaths of CaMV35S::GFP and CaMV35S::CTB4aGFP transgenic plants were used to determine the subcellular location. Root cells of CaMV35S::CTB4a-GFP transgenic plants stained with DiI were used for detection of fluorescence signals on the plasma membrane. CaMV35S:CTB4a-GFP vector and plasma membrane marker CD3-1007 (ref. 51) were transformed into Agrobacterium tumefaciens strain EHA105 and co-infiltrated into leaves of $N$. benthamiana with the suspension containing $10 \mathrm{mM}$ 2-(N-morpholino) ethanesulfonic acid, $10 \mathrm{mM} \mathrm{MgCl} 2$ and $150 \mu \mathrm{M}$ acetosyringone together with p19 (ref. 52) as previously described. After 3 days incubation at $25^{\circ} \mathrm{C}$, the tobacco leaves were used for fluorescence signal observation.

Green and red fluorescence were observed under a confocal microscope (OlympusFV1000). GFP was excited with a $488 \mathrm{~nm}$ laser, CD3-1007 and Dil were excited with a $543 \mathrm{~nm}$ laser. The emission spectra were collected at 500-550 nm for GFP, and 565-615 nm for CD3-1007 and Dil. To detect auto-fluorescence of chlorophyll, samples were examined with a long-pass $630 \mathrm{~nm}$ filter set.

Microscopy. For the morphological observations of anther, samples were fixed in FAA solution and then dehydrated through a graded ethanol and embedded in paraffin. Then $8-10 \mu \mathrm{m}$ thickness sections were obtained and stained using $0.025 \%$ toluidine blue for $30 \mathrm{~min}$, after which the washed sections were observed microscopically for photograph (Olympus SEX16). Pistils fixed with FAA solution were observed directly. For measuring pollen fertility, fixed anthers were ground to release pollen grains and then stained with $\mathrm{I}_{2}-\mathrm{KI}$ solution. Blue stained pollen grains were counted to determine pollen fertility.

For in situ hybridization experiment, the spikelets at about stage $10^{53}$ were collected and fixed in $4 \%(\mathrm{w} / \mathrm{v})$ paraformaldehyde at $4{ }^{\circ} \mathrm{C}$ overnight, followed by a series of dehydration by graded ethanol infiltration and finally were embedded in paraffin. Then $8 \mu \mathrm{m}$ thickness sections were obtained using microtome (Leica). 131 bp of CTB4a cDNA was sub-cloned into pMD19-T vector and used as template to generate antisense and sense probes. The digoxingenin labelled probes were prepared using digoxingenin RNA Labeling Kit (Roche, 1175025). After hybridization, the detection of digoxingenin-labelled was conducted using 
digoxingenin Nucleic Acid Detection Kit (Roche, 11175041910). The primer sequences used for probe preparation are given in Supplementary Data 2.

Haplotype analysis and SNP identification. DNA sequences of CTB4a in 137 rice accessions were amplified by PCR using primers listed in Supplementary Data 2. The PCR products were sequenced and used to perform haplotype analysis. Differences in phenotypic values and haplotypes were examined by one-way ANOVA or Student's $t$-tests. The Duncan multiple range test was conducted to make further comparisons if the results of the analyses were significant $(P<0.05)$. For one population sequence set a $47 \mathrm{~kb}$ segment, centred on CTB4a, was obtained from the rice $3 \mathrm{~K}$ project ${ }^{54}$ (RFGB, http://www.rmbreeding.cn/Index/s), with a missing rate of $\leq 80 \%$. A total of 295 indica, 144 japonica, 11 O. Rufipogon, 6 Aro and $3 \mathrm{Aus}$ accessions were retained for analysis. In addition, SNPs in the $47 \mathrm{~kb}$ region were used for the variety phylogenetic comparison of the CTB4a region.

Phylogenetic analysis of CTB4a. A neighbor-joining variety tree (1,000 replications of bootstrap tests) of rice varieties was constructed using MEGA 5.0 (ref. 55). The resulting tree was visualized and annotated using EvolView ${ }^{56}$. A total of 196 SNPs were used to analyse in the $7 \mathrm{~kb}$ region containing CTB4a. A panel of 448 rice and $11 O$. Rufipogon accessions was used to construct a minimum spanning tree for CTB4a. Arlequin version 3.5 (ref. 57) was used to define the haplotypes and to calculate the minimum spanning tree among haplotypes. Arlequin's distance matrix output was used in Hapstar-0.6 (ref. 58) to draw a minimum spanning tree.

Evaluation of artificial selection. The effects of artificial selection were evaluated according to the $\pi$ value of the $47 \mathrm{~kb}$ sequence. Nucleotide diversity was calculated by a custom PERL script. Different groups of rice accessions were examined including Tej, Tej-Hap-KMXBG, Tej-Hap-Towada, Trj, Ind and O. Rufipogon accessions. Statistical differences in averaged nucleotide diversity among the upstream $20 \mathrm{~kb}$ region, the middle $7 \mathrm{~kb}$ region including CTB4a and downstream $20 \mathrm{~kb}$ region were assessed for each subpopulation. Tajima' $D$ test of CTB4a in different groups was calculated and performed using DnaSP 5.10 (ref. 59). MLHKA $^{60}$ tests were used to detect the departure of CTB4a polymorphisms from neutrality with a set of known neutral genes, including Adhl, GBSSII, Ksl, Lhsl, Os0053, SSII1 and TFIIA $\gamma-1$ (ref. 33).

Transient expression in Arabidopsis protoplasts. For promoter activity analysis, a series of mutated pCTB4a::GUS fusion constructs were used for transient transformation into Arabidopsis protoplasts. The CaMV35S::LUC plasmid was used as an internal transformation control to provide an estimate of the extent of transient expression. The co-transferred protoplasts were incubated for $16 \mathrm{~h}$ at $25^{\circ} \mathrm{C}$ and $3 \mathrm{~h}$ at $4{ }^{\circ} \mathrm{C}$ before harvest. Sampled protoplasts were lysed in $100 \mu \mathrm{llysis}$ buffer (Promega, E4550). After centrifugation, the supernatant was used for glucuronidase (GUS) and firefly luciferase (Luc) activity analysis. GUS activity was assayed with $1 \mathrm{mM} 4$-methylumberlliferyl- $\beta$-d-glucuronide in lysis buffer. The reaction was terminated with $0.2 \mathrm{M} \mathrm{Na}_{2} \mathrm{CO}_{3}$ after $30 \mathrm{~min}$, the reaction product $\mathrm{MU}$ was measured using Fluorescence FLx800 microplate fluorescence reader (BIO-TEK Instruments). LUC activity was also determined by Fluorescence FLx800 microplate fluorescence reader. Ratios of GUS to LUC activities were used to define relative promoter activity. Three biological replicates, each with five technical replicates, were assayed for each construct. The cis-element analysis was conducted in PLACE (http://www.dna.affrc.go.jp/PLACE).

Yeast two-hybrid assay. In order to determine proteins interacting with CTB4a in rice, truncated cDNA of CTB4a containing a kinase domain $\left(C T B 4 \mathrm{a}^{\mathrm{KD}}\right)$ was amplified and sub-cloned into the pGBKT7 vector. CTB4 $\mathrm{a}^{\mathrm{KD}}$ protein without activation activity was used as bait to screen a cDNA library prepared from equal amounts of poly-(A) containing RNA from leaves and panicles after three days of cold stress at $16^{\circ} \mathrm{C}$. Experimental procedures for screening and plasmid isolation were performed according to the manufacturer's user guide (Clontech, PT3024-1). Yeast strain AH109 was used in this assay. Primer sequences are provided in Supplementary Data 2.

In vitro GST-pull down assay. In order to confirm the interaction between $\mathrm{CTB} 4 \mathrm{a}^{\mathrm{KD}}$ and AtpB in vitro, CTB4a ${ }^{\mathrm{KD}}$ and AtpB were amplified and sub-cloned into pGEX-4T-1 and pET28a, respectively. The plasmids were transformed into E.coli BL21. Then $5 \mu \mathrm{g}$ of purified CTB4a ${ }^{\mathrm{KD}}$-GST or GST and AtpB-His protein were incubated in $1 \mathrm{ml}$ of PBS buffer ( $\mathrm{pH} 7.4,0.1 \% \mathrm{NP}-40)$ at $4{ }^{\circ} \mathrm{C}$ with gentle agitation for $2 \mathrm{~h}$ before addition of $20 \mu \mathrm{l}$ of Glutathione Sepharose $4 \mathrm{~B}$ beads (GE healthcare, cat.no.17-0756-01), and continued incubation for $1 \mathrm{~h}$. The Glutathione Sepharose beads was collected by brief centrifugation, washed five times in PBS buffer, re-suspended in SDS loading buffer, subjected to SDS-PAGE electrophoresis, and probed with an anti-GST (Sigma, SAB5300159, dilution, 1:1,000) and anti-His antibody (Sigma, H1029, dilution, 1:2,000). The original western blot images are provided in Supplementary Fig. 25. Primer sequences are provided in Supplementary Data 2.
Co-immunoprecipitation assay. The Super::AtpB-Myc and CaMV35S::HF$\mathrm{CTB} 4 \mathrm{a}{ }^{\mathrm{KD}}$ plasmids were individually transformed into Agrobacterium tumefaciens strain EHA105. The strains were co-infiltrated into tobacco leaves with p19. Total proteins were extracted from tobacco leaves expressing Super::AtpB-Myc/ CaMV35S::HF-CTB4a ${ }^{\text {KD }}$ or Super:: AtpB-Myc/CaMV35S::HF using $300 \mu \mathrm{l}$ of IP buffer (50 mM Tris- $\mathrm{HCl}$, pH 7.5, $150 \mathrm{mM} \mathrm{NaCl}, 10 \mathrm{mM} \mathrm{MgCl} 2,0.1 \% \mathrm{NP}-40,3 \mathrm{mM}$ DTT and $1 \times$ Protease Inhibitor Cocktail, Roche). Lysates at $4{ }^{\circ} \mathrm{C}$ were centrifuged at $12,000 \mathrm{~g}$ for $20 \mathrm{~min}$ and $40 \mu \mathrm{l}$ was taken as input. The left supernatants were incubated with $20 \mu \mathrm{l} \mathrm{HA}$ agarose beads (Sigma, A2095) at $4{ }^{\circ} \mathrm{C}$ for at least $2 \mathrm{~h}$ in a $1 \mathrm{ml}$ medium supplemented with IP buffer. Subsequently, beads were collected by centrifugation at 5,000 $\mathrm{g}$ for $3 \mathrm{~min}$, washed five times with IP buffer and eluted with boiled $1 \times$ SDS loading buffer. Samples were then separated in SDS-PAGE gels and detected by anti-Myc (Sigma, M4439, dilution, 1:3,000) and anti-HA antibodies (Sigma, H3663, dilution, 1:3,000). The original western blot images are provided in Supplementary Fig. 25. Primer sequences are provided in Supplementary Data 2.

Determination of ATPase activity and ATP content. Panicles and leaves of different materials under normal $\left(28^{\circ} \mathrm{C}\right)$ and CS-PT for 3 days were sampled for determination of ATP synthase activity and ATP content. ATP synthase activity was determined by the protocol provided with the ATPase Activity Assay Kit (GenMed Scientifics, GMS50248.3). ATP was extracted in lysis buffer $(50 \mathrm{mM}$ Tris-HCl, pH 7.5, $100 \mathrm{mM} \mathrm{NaCl}, 1 \mathrm{mM}$ EDTA, $0.2 \%$ TritonX-100 and 2\% glycerol) and quantified based on the requirement of luciferase for ATP in producing light following the manufacturer's protocol supplied with the ATP Determination Kit (Invitrogen, A22066). A standard curve of ATP concentrations from $0.01 \mu \mathrm{M}$ to $3 \mu \mathrm{M}$ was used in the analysis. Each experiment was conducted with three biological samples, each with five technical replicates.

Measurement of photosynthetic rate. The photosynthetic rates of different materials under normal and CS-PT conditions were measured using a LICOR-6400 $\mathrm{CO}_{2}$ gas exchange analyser (LICOR-6400, Lincoln, NE). Statistical analysis was based on data obtained from at least eight plants of each line.

In vitro phosphorylation assay. The kinase activity assays of $\mathrm{CTB} 4 \mathrm{a}^{\mathrm{KD}}$ were carried out in a $15 \mu$ reaction mixture containing purified CTB4a ${ }^{\mathrm{KD}}$-GST $(2 \mu \mathrm{g}) /$ GST $(2 \mu \mathrm{g})$, CTB4a ${ }^{\mathrm{KD}}$-GST $(2 \mu \mathrm{g}) / \mathrm{AtpB}-\mathrm{GST}(2 \mu \mathrm{g})$ or GST $(2 \mu \mathrm{g}) / \mathrm{AtpB}-\mathrm{GST}$. The kinase buffer contained $50 \mathrm{mM}$ Tris- $\mathrm{HCl}(\mathrm{pH} 7.5), 10 \mathrm{mM} \mathrm{MgCl}_{2}$ and $20 \mathrm{mM}$ ATP. The reactions were started by adding $10 \mu \mathrm{Ci}$ of $\left[\gamma^{-}{ }^{32} \mathrm{P}\right]$ ATP and the samples were incubated for $30 \mathrm{~min}$ at $30^{\circ} \mathrm{C}$. The reaction was stopped by adding $3 \mu \mathrm{l}$ $6 \times$ loading buffer and boiled for $5 \mathrm{~min}$, then the proteins were separated by $10 \%$ SDS-polyacrylamide gel electrophoresis and detected by Typhoon 9410 imager (GE Healthcare). Primer sequences are provided in Supplementary Data 2.

Data availability. Accession code: the genomic DNA sequence of CTB4a in KMXBG has been deposited in GenBank with the accession number FJ693708.1.The authors declare that all data supporting the findings of this study are available within the manuscript and its supplementary files or are available from the corresponding author upon request.

\section{References}

1. Sasaki, T. \& Burr, B. International rice genome sequencing project: the effort to completely sequence the rice genome. Curr. Opin. Plant Biol. 3, 138-142 (2000).

2. Kuroki, M. et al. A quantitative trait locus for cold tolerance at the booting stage on rice chromosome 8. Theor. Appl. Genet. 115, 593-600 (2007).

3. Cruz, R. P. et al. Avoiding damage and achieving cold tolerance in rice plants. Food and Energy Security 2, 96-119 (2013).

4. Andaya, V. \& Mackill, D. QTLs conferring cold tolerance at the booting stage of rice using recombinant inbred lines from a japonica $\times$ indica cross. Theor. Appl. Genet. 106, 1084-1090 (2003).

5. Kaneda, C. \& Beachell, H. Breeding rice for cold tolerance. Saturday Seminar Paper 9 (International Rice Research Institute (IRRI), 1974).

6. Zhu, Y. et al. Identification and fine mapping of a stably expressed QTL for cold tolerance at the booting stage using an interconnected breeding population in rice. PLoS ONE 10, e0145704 (2015).

7. Zeng, Y. et al. QTLs of cold tolerance-related traits at the booting stage for NIL-RILs in rice revealed by SSR. Genes Genome 31, 143-154 (2009).

8. Takeuchi, Y. et al. Mapping quantitative trait loci controlling cool-temperature tolerance at booting stage in temperate japonica rice. Breeding Sci. 51, 191-197 (2001).

9. $\mathrm{Xu}, \mathrm{L}$. et al. Identification and mapping of quantitative trait loci for cold tolerance at the booting stage in a japonica rice near-isogenic line. Plant Sci. 174, 340-347 (2008).

10. Ye, C. et al. A QTL controlling low temperature induced spikelet sterility at booting stage in rice. Euphytica 176, 291-301 (2010). 
11. Zhou, L. et al. Fine mapping a QTL $q C T B 7$ for cold tolerance at the booting stage on rice chromosome 7 using a near-isogenic line. Theor. Appl. Genet. 121, 895-905 (2010).

12. Shirasawa, S., Endo, T., Nakagomi, K., Yamaguchi, M. \& Nishio, T. Delimitation of a QTL region controlling cold tolerance at booting stage of a cultivar,'Lijiangxintuanheigu', in rice, Oryza sativa L. Theor. Appl. Genet. 124, 937-946 (2012)

13. Cho, J. H. et al. QTLs analysis of agronomic traits based on cultivation of low and high altitude locations in rice (Oryza sativa L.). Korean J. Breeding Sci. 44, 110-120 (2012)

14. Pan, Y. et al. Genetic analysis of cold tolerance at the germination and booting stages in rice by association mapping. PLoS ONE 10, e0120590 (2015).

15. Endo, T. et al. Detection of QTLs for cold tolerance of rice cultivar 'Kuchum' and effect of QTL pyramiding. Theor. Appl. Genet. 129, 631-640 (2016).

16. Wang, D. et al. Genome-wide association mapping of cold tolerance genes at the seedling stage in rice. Rice 9, 61 (2016).

17. Yang, T. et al. Identification and pyramiding of QTLs for cold tolerance at the bud bursting and the seedling stages by use of single segment substitution lines in rice (Oryza sativa L.). Mol. Breeding 36, 1-10 (2016).

18. Miura, K., Lin, S., Yano, M. \& Nagamine, T. Mapping quantitative trait loci controlling low temperature germinability in rice (Oryza sativa L.). Breeding Sci. 51, 293-299 (2001).

19. Fujino, K. et al. Mapping of quantitative trait loci controlling low-temperature germinability in rice (Oryza sativa L.). Theor. Appl. Genet. 108, 794-799 (2004).

20. Andaya, V. \& Mackill, D. Mapping of QTLs associated with cold tolerance during the vegetative stage in rice. J. Exp. Bot. 54, 2579-2585 (2003).

21. Ma, Y. et al. COLD1 confers chilling tolerance in rice. Cell 160, 1209-1221 (2015).

22. Lu, G. et al. Rice LTG1 is involved in adaptive growth and fitness under low ambient temperature. Plant J. 78, 468-480 (2014).

23. Fujino, K. et al. Molecular identification of a major quantitative trait locus, qLTG3-1, controlling low-temperature germinability in rice. Proc. Natl Acad. Sci. USA 105, 12623-12628 (2008).

24. Saito, K., Hayano, Y., Kuroki, M. \& Sato, Y. Map-based cloning of the rice cold tolerance gene Ctb1. Plant Sci. 179, 97-102 (2010).

25. Dai, L. et al. Genetic analysis on cold tolerance characteristics of yunnan rice landrace ( Oryza sativa L. ) KUNMINGXIAOBAIGU. Chin. J. Rice Sci. 13, 73-76 (1999).

26. Dai, L., Ye, C., Kudo, S. \& Tanno, K. Research progress of cold tolerance in rice by fifteen years Sino-Japanese cooperation. China Seed Ind. 4, 40-42 (1998).

27. Satake, T. Male sterility caused by cooling treatment at the young microspore stage in rice plants. XXIX. The mechanism of enhancement in cool tolerance by raising water temperature before the critical stage. Jpn J. Crop Sci. 58, 240-245 (1989).

28. Sutoh, K. \& Yamauchi, D. Two cis-acting elements necessary and sufficient for gibberellin-upregulated proteinase expression in rice seeds. Plant J. 34, 635-645 (2003).

29. Mohanty, B., Krishnan, S. P. T., Swarup, S. \& Bajic, V. B. Detection and preliminary analysis of motifs in promoters of anaerobically induced genes of different plant species. Ann. Bot. 96, 669-681 (2005).

30. Nielsen, R., Hellmann, I., Hubisz, M., Bustamante, C. \& Clark, A. G. Recent and ongoing selection in the human genome. Nat. Rev. Genet. 8, 857-868 (2007).

31. Oleksyk, T. K., Smith, M. W. \& O’Brien, S. J. Genome-wide scans for footprints of natural selection. Philos. T. R. Soc. B Bio. Sci. 365, 185-205 (2010).

32. Tajima, F. Statistical method for testing the neutral mutation hypothesis by DNA polymorphism. Genetics 123, 585-595 (1989).

33. Zhu, Q., Zheng, X., Luo, J., Gaut, B. S. \& Ge, S. Multilocus analysis of nucleotide variation of Oryza sativa and its wild relatives: severe bottleneck during domestication of rice. Mol. Biol. Evol. 24, 875-888 (2007).

34. Dai, L., Ye, C., Yu, T. \& Xu, F. Studies on cold tolerance of rice, Oryza.sativa L. I. Description on types of cold injury and classifications of evaluation methods on cold tolerance in rice. Southwest China J. Agric. Sci. 15, 41-45 (2002).

35. Cui, S. et al. A proteomic analysis of cold stress responses in rice seedlings. Proteomics 5, 3162-3172 (2005).

36. Arbona, V., Manzi, M., Ollas, C. d. \& Gómez-Cadenas, A. Metabolomics as a tool to investigate abiotic stress tolerance in plants. Int. J. Mol. Sci. 14, 4885-4911 (2013).

37. Ruelland, E. \& Zachowski, A. How plants sense temperature. Environ. Exp. Bot. 69, 225-232 (2010).

38. Allen, D. J. \& Ort, D. R. Impacts of chilling temperatures on photosynthesis in warm-climate plants. Trends Plant Sci. 6, 36-42 (2001).

39. She, K. C. et al. Reduced rice grain production under high-temperature stress closely correlates with ATP shortage during seed development. Plant Biotechnol. 27, 67-73 (2010).

40. She, K. C., Yaeshima, M. \& Koumoto, T. High-temperature stress susceptibility of representative japonica rice cultivars derived from Norin-22: inadequate ATP supply during seed development may lead to severe damage. Plant Biotechnol. 29, 465-471 (2012).
41. Reiland, S. Large-scale Arabidopsis phosphoproteome profiling reveals novel chloroplast kinase substrates and phosphorylation networks. Plant Physiol. 150, 889-903 (2009).

42. Kanekatsu, M., Saito, H., Motohashi, K. \& Hisabori, T. The $\beta$ subunit of chloroplast ATP synthase $\left(\mathrm{CF}_{0} \mathrm{CF}_{1}\right.$-ATPase $)$ is phosphorylated by casein kinase II. IUBMB Life 46, 99-105 (1998).

43. Riego, G. D., Casano, L. M., Martín, M. \& Sabater, B. Multiple phosphorylation sites in the $\beta$ subunit of thylakoid ATP synthase. Photosynth. Res. 89, 11-18 (2006).

44. Irani, N. G. \& Russinova, E. Receptor endocytosis and signaling in plants. Curr Opin. Plant Biol. 12, 653-659 (2009).

45. Doebley, J. F., Gaut, B. S. \& Smith, B. D. The molecular genetics of crop domestication. Cell 127, 1309-1321 (2006).

46. Sang, T. \& Ge, S. Understanding rice domestication and implications for cultivar improvement. Curr. Opin. Plant Biol. 16, 139-146 (2013).

47. Jeong, D. H. et al. T-DNA insertional mutagenesis for activation tagging in rice. Plant Physiol. 130, 1636-1644 (2002).

48. Curtis, M. D. \& Grossniklaus, U. A gateway cloning vector set for highthroughput functional analysis of genes in planta. Plant Physiol. 133, 462-469 (2003).

49. Wang, Z. et al. A practical vector for efficient knockdown of gene expression in rice (Oryza sativa L.). Plant Mol. Biol. Rep. 22, 409-417 (2004).

50. Hiei, Y., Ohta, S., Komari, T. \& Kumashiro, T. Efficient transformation of rice (Oryza sativa L.) mediated by Agrobacterium and sequence analysis of the boundaries of the T-DNA. Plant J. 6, 271-282 (1994).

51. Nelson, B. K., Cai, X. \& Nebenführ, A. A multicolored set of in vivo organelle markers for co-localization studies in Arabidopsis and other plants. Plant J. 51, 1126-1136 (2007).

52. Lin, Q. et al. Rice APC/C $\mathrm{C}^{\mathrm{TE}}$ controls tillering by mediating the degradation of MONOCULM 1. Nat. comm. 3, 752 (2012).

53. Zhang, D. \& Wilson, Z. A. Stamen specification and anther development in rice. Sci. Bull. 54, 2342-2353 (2009).

54. Zheng, T. et al. Rice functional genomics and breeding database (RFGB): 3 K-rice SNP and InDel sub-database. Chin. Sci. Bull 60, 367-371 (2015).

55. Tamura, K. et al. MEGA5: molecular evolutionary genetics analysis using maximum likelihood, evolutionary distance, and maximum parsimony methods. Mol. Biol. Evol. 28, 2731-2739 (2011).

56. Zhang, H. et al. EvolView, an online tool for visualizing, annotating and managing phylogenetic trees. Nucleic Acids Res. 40, W569-W572 (2012).

57. Excoffier, L. \& Lischer, H. E. Arlequin suite ver 3.5: a new series of programs to perform population genetics analyses under Linux and Windows. Mol. Ecol. Resour. 10, 564-567 (2010).

58. Teacher, A. \& Griffiths, D. HapStar: automated haplotype network layout and visualization. Mol. Ecol. Resour. 11, 151-153 (2011).

59. Librado, P. \& Rozas, J. DnaSP v5: a software for comprehensive analysis of DNA polymorphism data. Bioinformatics 25, 1451-1452 (2009).

60. Wright, S. I. \& Charlesworth, B. The HKA test revisited: a maximumlikelihood-ratio test of the standard neutral model. Genetics 168, 1071-1076 (2004).

\section{Acknowledgements}

We thank Robert A. McIntosh (University of Sydney) and Jose M. Alonso (North Carolina State University) for critical reading and suggested revisions for the manuscript, S. Ge and Lian Zhou (Institute of Botany, Chinese Academy of Sciences) and X.M. Zheng (Institute of crop sciences, Chinese Academy of Agricultural Sciences) for the help with Tajima's $D$ values and MLHKA test analysis, Fengmei Li, Jinli Miao, Xin Wang and Yanfa Chen for preparing the samples, Xueqiang Wang, Yan Zhao and Xiaoyang Zhu for help with data analysis. This work was supported by grants from Ministry of Science and Technology of China (2016YFD0100300, 2015BAD02B01), the National Natural Science Foundation of China (31671649, 31471456), the Ministry of Agriculture of China (2014ZX08001-003-002 and 2014ZX08009-003-002), Chairman of Guangxi Autonomous Region (1517-03) and Key Program of Guangxi Academy of Agricultural Sciences (2016JZ05).

\section{Author contributions}

Z.Z., Jinjie Li and Z.L. designed the research, and together with Y.P., Jilong Li, L.Z. and H.S. performed most of experiments and analysed the data. Y.Z. constructed the NIL line and was responsible for cold tolerance evaluation under CS-HAA. H.G., Shuming Yang, W.Z., J.Y., X.S., G.L., Y.D., L.M., S.S. and L.D. performed part of the experiments. H.Z., Shuhua Yang and Y.G. conceived and supervised the project. Z.Z., Jinjie Li and Z.L. conceived the experiment and wrote the manuscript.

\section{Additional information}

Supplementary Information accompanies this paper at http://www.nature.com/ naturecommunications 
Competing interests: The authors declare no competing financial interest.

Reprints and permission information is available online at http://npg.nature.com/ reprintsandpermissions/

How to cite this article: Zhang, Z. et al. Natural variation in CTB4a enhances rice adaptation to cold habitats. Nat. Commun. 8, 14788 doi: 10.1038/ncomms14788 (2017).

Publisher's note: Springer Nature remains neutral with regard to jurisdictional claims in published maps and institutional affiliations. (c) (i) This work is licensed under a Creative Commons Attribution 4.0 International License. The images or other third party material in this article are included in the article's Creative Commons license, unless indicated otherwise in the credit line; if the material is not included under the Creative Commons license, users will need to obtain permission from the license holder to reproduce the material. To view a copy of this license, visit http://creativecommons.org/licenses/by/4.0/

(C) The Author(s) 2017 